\title{
Neural Network-Based Fault-Tolerant Control of Underactuated Surface Vessels
}

\author{
Bong Seok Park \\ Division of Electrical, Electronic, and Control Engineering, Kongju National University, Cheonan 31080, Republic of Korea \\ Correspondence should be addressed to Bong Seok Park; bspark@kongju.ac.kr
}

Received 1 September 2014; Accepted 27 October 2014

Academic Editor: Yudong Zhang

Copyright (C) 2015 Bong Seok Park. This is an open access article distributed under the Creative Commons Attribution License, which permits unrestricted use, distribution, and reproduction in any medium, provided the original work is properly cited.

This paper addresses the problem of trajectory tracking of underactuated surface vessels (USVs) in the presence of thruster failure. Multilayer neural networks (MNNs) are employed to estimate the unknown model parameters and external disturbances. To design a fault-tolerant controller without a fault detection scheme, we use the Nussbaum gain technique. We introduce an additional control to resolve the difficulty arising from having fewer inputs than degrees-of-freedom. Further, an approach angle is proposed to track both a straight and curved path. Stability analysis and simulations are performed to demonstrate the effectiveness of the proposed scheme.

\section{Introduction}

As land resources are depleted, interest in ocean exploration has increased. There are many marine vehicles designed to explore the ocean. Among these, unmanned marine vehicles (UMVs) such as autonomous underwater vehicles (AUVs) and unmanned surface vessels are useful because they can reduce the cost and the risk of personal injury [1]. Therefore, significant research has been undertaken to perform complex tasks using UMVs.

An important problem in the control of UMVs is failure occurrence on the actuators $[2,3]$. If there is no immediate solution for actuator failure, the UMV must abort the mission and return to the base for repair. This results in a waste of time and money. Therefore, to enhance the reliability while performing its mission in the presence of thruster failure, there are more thrusters than the degrees-of-freedom of the UMV. For this reason, the thruster-force allocation problem is important to overcome thruster failure [4]. The allocation of thruster forces of an AUV that can accommodate both thruster faults and saturation was presented in $[5,6]$. A fault diagnosis and accommodation system was proposed using weighted pseudoinverse in [7]. Weighted pseudoinverse and quantum particle swarm optimization were introduced for hybrid fault-tolerant control in [8]. The combination of backstepping technique and fuzzy control was presented for tracking control of fully actuated surface vessels in [9]. However, all these works focused on fully or overactuated systems. This implies that the cost and weight of the system are increased because there must be more devices. Moreover, if a fully actuated system is damaged, we then have an underactuated controller [10]. Therefore, it is necessary to develop a fault-tolerant controller for underactuated systems.

Motivated by these observations, a neural network-based fault-tolerant control is presented for underactuated surface vessels (USVs). The proposed algorithm can be applied to AUVs because the equations of motion for surface vessels are the same as those of AUVs in the horizontal plane. For a practical perspective, we assume that the bow and stern are not symmetric and the model parameters for the hydrodynamic terms are unknown. To estimate the unknown model parameters and external disturbances, we employ multilayer neural networks (MNNs) [11]. Unlike other fault-tolerant control methods [12-14], we do not require a fault detection scheme; we design the fault-tolerant controller using the Nussbaum gain technique [15]. Further, an additional control is introduced to resolve the difficulty of controller design of underactuated systems, and an approach angle is proposed to track any trajectory including both a straight- and curved-line path. It is proved that all 
the signals of the closed-loop system are ultimately, uniformly bounded. Simulation results are presented to demonstrate the effectiveness of the proposed scheme.

The main contributions of our paper are as follows. (1) The fault-tolerant controller is designed without the fault detection scheme. (2) For underactuated surface vessels, we develop the additional control input and analyze the stability including the sway dynamics. (3) The approach angle which is composed only of position information of USVs is introduced. With the help of a novel approach angle, we can track any trajectory including both a straight- and curved-line path. (4) For a practical application, the model parameters for the hydrodynamic terms and external disturbances are assumed to be unknown. These uncertainties are estimated by MNNs.

Throughout this paper, the following notations are used: (1) $\|\cdot\|$ denotes any suitable vector norm; (2) $\operatorname{tr}(\cdot)$ is the trace; (3) $\|\cdot\|_{F}$ is the Frobenius norm defined by $\|A\|_{F}=\sqrt{\operatorname{tr}\left(A^{T} A\right)}$; (4) $W_{i}$ and $\widehat{W}_{i}$ denote bounded ideal neural weights and the estimates of neural weights, respectively; (5) $\widetilde{W}_{i}=W_{i}-$ $\widehat{W}_{i}$ denotes the error between $W_{i}$ and $\widehat{W}_{i}$; (6) $\lambda_{\text {min }}(A)$ and $\lambda_{\text {max }}(A)$ denote the minimum and maximum eigenvalues of matrix $A$, respectively; (7) $\operatorname{diag}(\cdot)$ is a diagonal matrix.

\section{Underactuated Surface Vessel Model}

The kinematics and dynamics of USVs are described as follows [16]:

$$
\begin{gathered}
\dot{\eta}=J(\psi) \nu, \\
M \dot{v}=-C(\nu) \nu-D(\nu) \nu+\tau_{d}+\tau,
\end{gathered}
$$

where $\eta=[x, y, \psi]^{T}$ denotes position $(x, y)$ and yaw angle $\psi$ of the USV in the earth-fixed frame; $v=[u, v, r]^{T}$ denotes surge, sway, and yaw velocities of the USV in the body-fixed frame, respectively, $\tau_{d}=\left[\tau_{d, u}, \tau_{d, v}, \tau_{d, r}\right]^{T}$ denotes the external forces such as wind and ocean currents, and $\tau=\left[\tau_{u}, 0, \tau_{r}\right]^{T}$ is the control vector of the surge force $\tau_{u}$ and the yaw moment $\tau_{r}$. In the above equation, matrices $J(\psi), D(\nu), C(\nu)$, and $M$ are given as follows:

$$
\begin{aligned}
J(\psi) & =\left[\begin{array}{ccc}
\cos \psi & -\sin \psi & 0 \\
\sin \psi & \cos \psi & 0 \\
0 & 0 & 1
\end{array}\right], \\
D(\nu) & =\left[\begin{array}{ccc}
d_{11}(u) & 0 & 0 \\
0 & d_{22}(v, r) & d_{23}(v, r) \\
0 & d_{32}(v, r) & d_{33}(v, r)
\end{array}\right], \\
C(\nu) & =\left[\begin{array}{ccc}
0 & 0 & -m_{22} v-m_{t} r \\
0 & 0 & m_{11} u \\
m_{22} v+m_{t} r & -m_{11} u & 0
\end{array}\right], \\
M & =\left[\begin{array}{ccc}
m_{11} & 0 & 0 \\
0 & m_{22} & m_{23} \\
0 & m_{32} & m_{33}
\end{array}\right],
\end{aligned}
$$

where

$$
\begin{gathered}
m_{11}=m-X_{\dot{u}}, \quad m_{22}=m-Y_{\dot{v}}, \quad m_{23}=m x_{g}-Y_{\dot{r}} \\
m_{32}=m x_{g}-N_{\dot{v}}, \quad m_{33}=I_{z}-N_{\dot{r}} \\
m_{t}=\frac{\left(m_{23}+m_{32}\right)}{2}, \quad d_{11}(u)=-\left(X_{u}+X_{u|u|}|u|\right) \\
d_{22}(v, r)=-\left(Y_{v}+Y_{|v| v}|v|+Y_{|r| v}|r|\right) \\
d_{23}(v, r)=-\left(Y_{r}+Y_{|v| r}|v|+Y_{|r| r}|r|\right) \\
d_{32}(v, r)=-\left(N_{v}+N_{|v| v}|v|+N_{|r| v}|r|\right) \\
d_{33}(v, r)=-\left(N_{r}+N_{|v| r}|v|+N_{|r| r}|r|\right)
\end{gathered}
$$

$X_{u}, X_{u|u|}, Y_{v}, Y_{|v| v}, Y_{|r| v}, Y_{r}, Y_{|v| r}, Y_{|r| r}, N_{v}, N_{|v| v}, N_{|r| v}, N_{r}, N_{|v| r}$, and $N_{|r| r}$ are the linear and quadratic drag coefficients, $m$ is the mass of the USV, $X_{\dot{u}}, Y_{\dot{v}}, Y_{\dot{r}}, N_{\dot{v}}$, and $N_{\dot{r}}$ are the added masses, $x_{q}$ is the $X_{b}$-coordinate of the USV center of gravity in the body-fixed frame, $I_{z}$ is the inertia with respect to the vertical axis, and $M$ is invertible.

Remark 1. Unlike other works, we assume that the parameters $m_{23}$ and $m_{32}$ are not zero. This means that the sway dynamics is influenced by the yaw moment $\tau_{r}$ and we must control sway and yaw velocities simultaneously using only the yaw moment $\tau_{r}$. When the parameters $m_{23}$ and $m_{32}$ are not zero, controller design difficulties are increased.

To address the problem stated in Remark 1, we utilize the following state transformations [17]:

$$
\bar{x}=x+\epsilon \cos \psi, \quad \bar{y}=y+\epsilon \sin \psi, \quad \bar{v}=v+\epsilon r,
$$

where $\epsilon=m_{23} / m_{22}$. The transformed equation (4) implies that the virtual center of mass is positioned by $\epsilon$ in the longitudinal direction. Using (4), the USV dynamics (1) can be expressed by defining state variables $z_{1}=\left[z_{11}, z_{12}, z_{13}\right]^{T}=$ $[\bar{x}, \bar{y}, \psi]^{T}$ and $z_{2}=\left[z_{21}, z_{22}, z_{23}\right]^{T}=[u, \bar{v}, r]^{T}$ :

$$
\begin{aligned}
& \dot{z}_{1}=J(\psi) z_{2}, \\
& \dot{z}_{2}=\varphi\left(z_{2}\right)+\bar{\tau} \\
& y=z_{1},
\end{aligned}
$$

where

$$
\begin{gathered}
\varphi\left(z_{2}\right)=\left[\varphi_{1}, \varphi_{2}, \varphi_{3}\right]^{T}, \\
\varphi_{1}=\frac{\left(m_{22} v r+m_{t} r^{2}-d_{11}(u) u+\tau_{d, u}\right)}{m_{11}} \\
\varphi_{2}=-\frac{m_{11}}{m_{22}} u r-\frac{d_{22}(v, r)}{m_{22}} v-\frac{d_{23}(v, r)}{m_{22}} r+\frac{1}{m_{22}} \tau_{d, v}
\end{gathered}
$$




$$
\begin{gathered}
\varphi_{3}=\frac{1}{\Delta}\left\{\left(m_{11} m_{22}-m_{22}^{2}\right) u v+\left(m_{11} m_{32}-m_{t} m_{22}\right) u r\right. \\
-\left(d_{33}(v, r) r+d_{32}(v, r) v\right) m_{22} \\
+\left(d_{23}(v, r) r+d_{22}(v, r) v\right) m_{32} \\
\left.-m_{32} \tau_{d, v}+m_{22} \tau_{d, r}\right\} \\
\Delta=m_{22} m_{33}-m_{23} m_{32}, \\
\bar{\tau}=\left[\bar{\tau}_{1}, 0, \bar{\tau}_{2}\right]^{T}=\left[\frac{\tau_{u}}{m_{11}}, 0, \frac{m_{22} \tau_{r}}{\Delta}\right]^{T} .
\end{gathered}
$$

In these expressions, we assume that $\varphi_{u}, \varphi_{v}$, and $\varphi_{r}$ are unknown time-varying functions because they cannot be obtained precisely by measurement or calculation.

When faults occur in the control vector $\bar{\tau}$, we can express them as follows:

$$
\bar{\tau}=F_{p} \tau_{n}+f_{b}
$$

where $\tau_{n}=\left[\tau_{n, 1}, 0, \tau_{n, 2}\right]^{T}$ is a nominal control vector, $F_{p}=$ $\operatorname{diag}\left(f_{1}, 0, f_{2}\right) \in \mathbb{R}^{3 \times 3}$ denotes the partial loss of control vector, and $f_{b}=\left[f_{b, 1}, 0, f_{b, 2}\right]^{T}$ is an unknown bias vector. $f_{1}$ and $f_{2}$ are defined as

$$
f_{i}= \begin{cases}0<f_{i}<1 ; & \text { if the thrusters are in partial failure } \\ f_{i}=1 ; & \text { if the thrusters are not in failure. }\end{cases}
$$

The control objective is to design a fault-tolerant controller such that it tracks the reference trajectory $\eta_{d}=$ $\left[x_{d}, y_{d}, \psi_{d}\right]$ even though faults exist in the control vector of the USV. The reference trajectory is given as

$$
\begin{aligned}
& \dot{x}_{d}=u_{d} \cos \psi_{d}-v_{d} \sin \psi_{d}, \\
& \dot{y}_{d}=u_{d} \sin \psi_{d}+v_{d} \cos \psi_{d}, \\
& \dot{\psi}_{d}=r_{d},
\end{aligned}
$$

where $x_{d}$ and $y_{d}$ denote the desired position; $\psi_{d}$ denotes the desired yaw angle, and $u_{d}, v_{d}$, and $r_{d}$ are the desired velocities of the USV. To generate the reference trajectory, we can use path planning techniques presented in $[18,19]$.

Assumption 1. The desired velocities $u_{d}, v_{d}$, and $r_{d}$ are bounded. Further, the first derivatives $\dot{u}_{d}, \dot{v}_{d}$, and $\dot{r}_{d}$ are available and bounded.

\section{Fault-Tolerant Control}

3.1. Neural Network Estimation. In this paper, MNN is used to estimate the unknown function $\varphi\left(z_{2}\right)$. From the universal approximation property for neural networks (NNs) [20], the nonlinear function $\varphi\left(z_{2}\right)$ can be written as

$$
\varphi\left(z_{2}\right)=W^{T} \sigma\left(V^{T} z_{2}\right)+\varepsilon,
$$

where $\varepsilon$ is the $\mathrm{NN}$ reconstruction error and $\sigma(\cdot)$ is the sigmoid function defined as $\sigma(z)=1 /\left(1+e^{-z}\right)$.
Assumption 2. $W$ and $V$ are bounded such that $\|W\|_{F} \leq$ $W_{M}$ and $\|V\|_{F} \leq V_{M}$ for positive constants $W_{M}$ and $V_{M}$, respectively, and $\varepsilon$ is bounded such that $\|\varepsilon\| \leq \varepsilon_{M}$ for a positive constant $\varepsilon_{M}$.

The Taylor series expansion of $\sigma(\cdot)$ in (10) for a given $z_{2}$ can be written as

$$
\sigma\left(V^{T} z_{2}\right)=\sigma\left(\widehat{V}^{T} z_{2}\right)+\bar{\sigma}\left(\widehat{V}^{T} z_{2}\right) \widetilde{V}^{T} z_{2}+H\left(\widetilde{V}^{T} z_{2}\right)^{2}
$$

where $\bar{\sigma}=\left.\left(d \sigma\left(V^{T} z_{2}\right) / d\left(V^{T} z_{2}\right)\right)\right|_{V^{T} z_{2}=\widehat{V}^{T} z_{2}}, H\left(\widetilde{V}^{T} z_{2}\right)^{2}$ denotes higher order terms, and $\widetilde{V}=V-\widehat{V}$. From (10) and (11), we can obtain the following result:

$$
\varphi\left(z_{2}\right)-\widehat{\varphi}\left(z_{2}\right)=\widetilde{W}^{T} \sigma\left(\widehat{V}^{T} z_{2}\right)+\widehat{W}^{T} \bar{\sigma}\left(\widehat{V}^{T} z_{2}\right) \widetilde{V}^{T} z_{2}+P
$$

where $P=\widetilde{W}^{T} \bar{\sigma}\left(\widehat{V}^{T} z_{2}\right) \widetilde{V}^{T} z_{2}+W^{T} H\left(\widetilde{V}^{T} z_{2}\right)^{2}+\varepsilon$.

Property 1. The unknown term $P$ in (12) is bounded such that $\|P\| \leq \delta^{T} \Theta$, where $\delta=\left[c_{0}, c_{1}, c_{2}, c_{3}, c_{4}\right]^{T}, \Theta=\left[1,\left\|z_{2}\right\|\right.$, $\left.\|\widehat{W}\|_{F}\left\|z_{2}\right\|,\|\widehat{V}\|_{F}\left\|z_{2}\right\|,\|\widehat{W}\|_{F}\|\widehat{V}\|_{F}\left\|z_{2}\right\|\right]^{T}$, and $c_{j}(j=0, \ldots, 4)$ is a positive bounding constant.

Proof. See the appendix.

3.2. Neural Network-Based Fault-Tolerant Control. In this section, we design the neural network-based fault-tolerant control system. To avoid the difficulty of detecting the faults, the controller is proposed using the Nussbaum gain technique. The following lemma is used to design the neural network-based fault-tolerant control law.

Lemma 2 (see [21]). Let $V(\cdot)$ and $\zeta(\cdot)$ be smooth functions defined on $\left[0, t_{f}\right)$ with $V(t) \geq 0, \forall t \in\left[0, t_{f}\right)$. For $t \in\left[0, t_{f}\right)$, if the following inequality holds,

$$
V(t) \leq \alpha_{2}+e^{-\alpha_{3} t} \int_{0}^{t} g(\lambda) N(\zeta) \dot{\zeta}^{\alpha_{3} \lambda} d \lambda+e^{-\alpha_{3} t} \int_{0}^{t} \dot{\zeta}^{\alpha_{3} \lambda} d \lambda,
$$

where $\alpha_{2}$ and $\alpha_{3}$ are positive constants, $N(\zeta)=e^{\zeta^{2}} \cos ((\pi / 2) \zeta)$ is a Nussbaum-type function, and $g(t)$ is a time-varying parameter which takes values in the closed intervals $\Omega:=$ $\left[\omega^{-}, \omega^{+}\right]$, then $V(t), \zeta(t)$, and $\int_{0}^{t} g(\lambda) N(\zeta) \dot{\zeta} d \lambda$ must be bounded on $\left[0, t_{f}\right)$.

We now design the neural network-based fault-tolerant control law using the dynamic surface design approach.

Step 1. Define the following errors:

$$
\begin{aligned}
& e_{1}=z_{11}-\bar{x}_{d}, \\
& e_{2}=z_{12}-\bar{y}_{d}, \\
& e_{3}=z_{13}-\psi_{a},
\end{aligned}
$$


where $\bar{x}_{d}=x_{d}+\epsilon \cos \psi_{d}$ and $\bar{y}_{d}=y_{d}+\epsilon \sin \psi_{d}$. An approach angle $\psi_{a}$ is defined as

$$
\psi_{a}=\arctan \left(\frac{e_{2}}{e_{1}}\right) \tanh \left(\frac{D^{2}}{\gamma_{1}}\right)+\psi_{d}\left(1-\tanh \left(\frac{D^{2}}{\gamma_{1}}\right)\right)
$$

where $D=\sqrt{e_{1}^{2}+e_{2}^{2}}$ and $\gamma_{1}$ is a positive constant.

Remark 3. In this paper, we use an approach angle $\psi_{a}$ as an alternative for the desired yaw angle $\psi_{d}$. Using the approach angle $\psi_{a}$, we can track any trajectory including both straightline and curved-line paths. There are other works using the approach angle; however, our approach angle is different from the other studies because it requires only position information.

Using (5) and (9), the time derivative of (14) is given as

$$
\begin{aligned}
& \dot{e}_{1}=z_{21} \cos z_{13}-z_{22} \sin z_{13}-u_{d} \cos \psi_{d}+\bar{v}_{d} \sin \psi_{d}, \\
& \dot{e}_{2}=z_{21} \sin z_{13}+z_{22} \cos z_{13}-u_{d} \sin \psi_{d}-\bar{v}_{d} \cos \psi_{d}, \\
& \dot{e}_{3}=z_{23}-\dot{\psi}_{a} .
\end{aligned}
$$

To stabilize the error dynamics of (16), we choose the virtual controls $\phi_{1}, \phi_{2}$, and $\phi_{3}$ as

$$
\begin{aligned}
\phi_{1}= & -k_{1} e_{1} \cos z_{13}-k_{1} e_{2} \sin z_{13} \\
& +u_{d} \cos \left(z_{13}-\psi_{d}\right)+\bar{v}_{d} \sin \left(z_{13}-\psi_{d}\right), \\
\phi_{2}= & k_{1} e_{1} \sin z_{13}-k_{1} e_{2} \cos z_{13} \\
& -u_{d} \sin \left(z_{13}-\psi_{d}\right)+\bar{v}_{d} \cos \left(z_{13}-\psi_{d}\right), \\
\phi_{3}= & -k_{2} e_{3}+\dot{\psi}_{a},
\end{aligned}
$$

where $k_{1}$ and $k_{2}$ are design parameters.

Step 2. In this step, we use the NNs to estimate uncertain terms $\varphi_{1}, \varphi_{2}$, and $\varphi_{3}$. From (12), we can derive the following results:

$$
\begin{array}{r}
\varphi_{i}-\widehat{\varphi}_{i}=\widetilde{W}_{i}^{T} \sigma\left(\widehat{V}_{i} z_{2}\right)+\widehat{W}_{i}^{T} \bar{\sigma}\left(\widehat{V}_{i}^{T} z_{2}\right) \widetilde{V}_{i}^{T} z_{2}+P_{i}, \\
i=1,2,3,
\end{array}
$$

where $\widehat{\varphi}_{i}$ is the estimate of $\varphi_{i}$ and $P_{i}=\widetilde{W}_{i}^{T} \bar{\sigma}\left(\widehat{V}_{i}^{T} z_{2}\right) \widetilde{V}_{i}^{T} z_{2}+$ $W_{i}^{T} H\left(\widetilde{V}_{i}^{T} z_{2}\right)^{2}+\varepsilon_{i}$. By Property $1, P_{i}$ is bounded such that $\left\|P_{i}\right\| \leq \delta_{i}^{T} \Theta_{i}$, where $\delta_{i}$ is the unknown constant vector and $\Theta_{i}=\left[1,\left\|z_{2}\right\|,\left\|\widehat{W}_{i}\right\|\left\|z_{2}\right\|,\left\|\widehat{V}_{i}\right\|\left\|z_{2}\right\|,\left\|\widehat{W}_{i}\right\|\left\|\widehat{V}_{i}\right\|\left\|z_{2}\right\|\right]^{T}$.

Assumption 3. $W_{i}$ and $V_{i}$ are bounded such that $\left\|W_{i}\right\| \leq$ $W_{i, M}$ and $\left\|V_{i}\right\| \leq V_{i, M}$ for positive constants $W_{i, M}$ and $V_{i, M}$, respectively, and $\varepsilon_{i}$ is bounded such that $\left\|\varepsilon_{i}\right\| \leq \varepsilon_{i, M}$ for a positive constant $\varepsilon_{i, M}$.
Define the following errors:

$$
\begin{aligned}
& e_{4}=z_{21}-\phi_{1, f}-\gamma_{2} \tanh \beta_{1}, \\
& e_{5}=z_{22}-\phi_{2, f}-\gamma_{2} \tanh \beta_{2}, \\
& e_{6}=z_{23}-\phi_{3, f}-\gamma_{2} \tanh \beta_{3},
\end{aligned}
$$

where $\gamma_{2}$ is a positive constant. $\phi_{1, f}, \phi_{2, f}$, and $\phi_{3, f}$ are signals obtained by the following first-order filters:

$$
\kappa_{i} \dot{\phi}_{i, f}+\phi_{i, f}=\phi_{i}, \quad \phi_{i, f}(0)=\phi_{i}(0), \quad i=1,2,3,
$$

where $\kappa_{i}$ is a positive constant. Here, $\beta_{1}, \beta_{2}$, and $\beta_{3}$ are used to solve the problem that there is no actual control on the sway dynamics as seen from (5) and obtained by the following differential equations:

$$
\begin{aligned}
& \dot{\beta}_{1}=-\frac{\cosh ^{2} \beta_{1}}{\gamma_{2}} T_{u} \tanh \beta_{2}, \\
& \dot{\beta}_{2}=\frac{\cosh ^{2} \beta_{2}}{\gamma_{2}}\left(k_{3} e_{5}-e_{1} \sin z_{13}+e_{2} \cos z_{13}+\widehat{\varphi}_{2}\right. \\
& \left.\quad+\widehat{\delta}_{2}^{T} \Theta_{2} \operatorname{sgn}\left(e_{5}\right)-\dot{\phi}_{2, f}\right), \\
& \dot{\beta}_{3}=-\frac{\cosh ^{2} \beta_{3}}{\gamma_{2}} T_{r} \tanh \beta_{2},
\end{aligned}
$$

where $T_{u}, T_{r}, k_{3}>0$ are design parameters and $\widehat{\delta}_{2}$ is the estimate of $\delta_{2}$. To design the unified control algorithm from the error dynamics of (19), we introduce a function $h(\rho(t))$ as

$$
h(\rho(t))= \begin{cases}1, & \|\widetilde{y}(t)\|>\rho(t) \\ 0, & \|\widetilde{y}(t)\| \leq \rho(t),\end{cases}
$$

where $\rho(t)$ is the threshold which is the criterion for judgment.

Remark 4. The threshold $\rho(t)$ can be derived from the fault detection algorithm. However, the proposed algorithm does not require the threshold $\rho(t)$ because we design the neural network-based fault-tolerant controller under the assumption that the function $h(\rho(t))$ in (22) is unknown.

Using (5), (7), and (22), the time derivative of (19) is represented as

$$
\begin{gathered}
\dot{e}_{4}=\bar{\varphi}_{1}+g_{1}(t) \tau_{n, 1}+T_{u} \tanh \beta_{2}-\dot{\phi}_{1, f}, \\
\dot{e}_{5}=\varphi_{2}-k_{4} e_{5}+e_{1} \sin z_{13}-e_{2} \cos z_{13} \\
\quad-\widehat{\varphi}_{2}-\widehat{\delta}_{2}^{T} \Theta_{2} \operatorname{sgn}\left(e_{5}\right), \\
\dot{e}_{6}=\bar{\varphi}_{3}+g_{2}(t) \tau_{n, 2}+T_{r} \tanh \beta_{2}-\dot{\phi}_{3, f},
\end{gathered}
$$

where

$$
\begin{gathered}
\bar{\varphi}_{1}=\varphi_{1}+h(\rho(t)) f_{b, 1}, \\
\bar{\varphi}_{3}=\varphi_{3}+h(\rho(t)) f_{b, 2}, \\
g_{1}(t)=1-h(\rho(t))+h(\rho(t)) f_{1}, \\
g_{2}(t)=1-h(\rho(t))+h(\rho(t)) f_{2} .
\end{gathered}
$$


Property 2. $g_{1}(t)$ and $g_{2}(t)$ are bounded such that $0<g_{1}(t) \leq$ 1 and $0<g_{2}(t) \leq 1$.

Proof. See the appendix.

From (23), we choose the actual controls $\tau_{n, 1}$ and $\tau_{n, 2}$ as follows:

$$
\begin{gathered}
\tau_{n, 1}=N\left(\zeta_{1}\right) \theta_{1}, \\
\theta_{1}=\widehat{\bar{\varphi}}_{1}+k_{3} e_{4}-\dot{\phi}_{1, f}+T_{u} \tanh \beta_{2}+e_{1} \cos z_{13} \\
+e_{2} \sin z_{13}+\widehat{\delta}_{1}^{T} \Theta_{1} \operatorname{sgn}\left(e_{4}\right), \\
\dot{\zeta}_{1}=e_{4} \theta_{1}, \\
\tau_{n, 2}=N\left(\zeta_{2}\right) \theta_{2}, \\
\theta_{2}=\widehat{\bar{\varphi}}_{3}+k_{3} e_{6}-\dot{\phi}_{3, f}+T_{r} \tanh \beta_{2}+\widehat{\delta}_{3}^{T} \Theta_{3} \operatorname{sgn}\left(e_{6}\right)+e_{3}, \\
\dot{\zeta}_{2}=e_{6} \theta_{2},
\end{gathered}
$$

where $N\left(\zeta_{1}\right)$ and $N\left(\zeta_{2}\right)$ are Nussbaum-type functions. For the stability analysis, we define the boundary layer error as

$$
\omega_{i}=\phi_{i, f}-\phi_{i}, \quad i=1,2,3 .
$$

Let us consider the following Lyapunov functions:

$$
\begin{aligned}
& V_{1}=\frac{1}{2}\left\{e_{1}^{2}\right.+e_{2}^{2}+e_{4}^{2}+e_{5}^{2}+\sum_{i=1}^{2} \omega_{i}^{2} \\
&\left.\quad+\sum_{i=1}^{2}\left(\widetilde{W}_{i}^{T} \Lambda_{i}^{-1} \widetilde{W}_{i}+\operatorname{tr}\left(\widetilde{V}_{i}^{T} \Lambda_{i}^{-1} \widetilde{V}_{i}\right)+\widetilde{\delta}_{i}^{T} \Lambda_{i}^{-1} \widetilde{\delta}_{i}\right)\right\}, \\
& V_{2}=\frac{1}{2}\left(e_{3}^{2}+e_{6}^{2}+\omega_{3}^{2}\right. \\
&\left.\quad+\widetilde{W}_{3}^{T} \Lambda_{3}^{-1} \widetilde{W}_{3}+\operatorname{tr}\left(\widetilde{V}_{3}^{T} \Lambda_{3}^{-1} \widetilde{V}_{3}\right)+\widetilde{\delta}_{3}^{T} \Lambda_{3}^{-1} \widetilde{\delta}_{3}\right),
\end{aligned}
$$

where $\Lambda_{i}$ is positive definite matrix.

Theorem 5. Consider the USV model (5) in the presence of the faults. For any initial conditions $V_{1}(0) \leq \mu_{1}$ and $V_{2}(0) \leq \mu_{2}$, where $\mu_{1}$ and $\mu_{2}$ are any positive constants, if Assumptions 1-3 are satisfied and one applies the controllers (17) and (25) with the adaptation laws defined as

$$
\begin{gathered}
\widehat{W}_{i}=\Lambda_{i} e_{i+3} \sigma\left(\widehat{V}_{i}^{T} z_{2}\right)-\iota_{i} \Lambda_{i} \widehat{W}_{i}, \\
\widehat{V}_{i}=\Lambda_{i} z_{2}\left(\bar{\sigma}\left(\widehat{V}_{i}^{T} z_{2}\right) \widehat{W}_{i} e_{i+3}\right)^{T}-\iota_{i} \Lambda_{i} \widehat{V}_{i}, \\
\dot{\widehat{\delta}}_{i}=\Gamma_{i} \Theta_{i}\left|e_{i+3}\right|-\iota_{i} \Gamma_{i} \widehat{\delta}_{i}, \\
i=1,2,3,
\end{gathered}
$$

where $t_{i}$ is a positive constant, then one can ensure that

$$
\lim _{t \rightarrow \infty}\left\|\eta-\eta_{d}\right\|=\vartheta
$$

where $\vartheta$ is a positive constant.

Proof. See the appendix.

\section{Simulation Results}

In this section, we simulate a neural network-based faulttolerant controller to verify the performance of the proposed control system. The parameters of the underactuated surface vessel are as indicated in [22]. The control gains are chosen as $k_{1}=1.2, k_{2}=k_{3}=2, T_{u}=T_{r}=1, \gamma_{1}=0.0001, \gamma_{2}=1000$, and $\kappa_{i}=0.2$, where $i=1,2,3$. The reference trajectory is generated by the following desired velocities:

$$
\begin{gathered}
0 \leq t<5:\left[u_{d}, v_{d}, r_{d}\right]=\left[0.5 \sin \left(\frac{\pi t}{10}\right), 0,0\right], \\
5 \leq t<20:\left[u_{d}, v_{d}, r_{d}\right]=[0.5,0,0], \\
20 \leq t:\left[u_{d}, v_{d}, r_{d}\right]=[0.5,0,0.3] .
\end{gathered}
$$

The initial postures for the reference USV and the actual USV are $\left[x_{d}, y_{d}, \psi_{d}\right]=[0,0,0]$ and $[x, y, \psi]=[-0.5,0.5,0]$, respectively. In this simulation, we use three MNNs to estimate the uncertainties and the MNNs are composed of four hidden layers and three inputs. In addition, the weight tuning parameters of the MNNs are chosen as $\iota_{i}=0.01, \Lambda_{i}=$ $\operatorname{diag}(2,2,2)$, and $\Gamma_{i}=\operatorname{diag}(0.001,0.001,0.001,0.001,0.001)$, where $i=1,2,3$. To verify the robustness of the proposed neural network-based fault-tolerance controller, we assume that the faults occur after 20 seconds such that $F_{p}=$ $\operatorname{diag}(0.6,0,0.5)$ and $f_{b}=[1.5,0,1]$.

Figure 1(a) presents the trajectory tracking result. The tracking errors are depicted in Figures 1(c) and 1(d). As indicated in Figures 1(c) and 1(d), there are tracking errors when faults occur on $t=20(\mathrm{sec})$; however these errors are reduced quickly. Further, the tracking errors are bounded for the entire time even though faults exist. From Figure 1(a), we can confirm that the actual USV tracks successfully the reference trajectory including both straight and curved paths. Therefore, we can conclude that the proposed control system possesses robustness for faults, model uncertainties, and external disturbances.

\section{Conclusions}

This paper presented a neural network-based fault-tolerant controller for an underactuated USV with faults, model uncertainties, and external disturbances. MNNs were employed to estimate the highly nonlinear uncertain terms, and approach angle was implemented to track any reference trajectory including both straight- and curved-line paths. To avoid the difficulty of detecting the faults, the neural network-based fault-tolerant controller was designed using the Nussbaum gain technique. From the simulation results, it was demonstrated that the proposed control system has good tracking performance in the presence of the faults, model uncertainties, and external disturbances. In the future, the actuator saturation problem will be addressed and evaluate the performance through real experiments. 


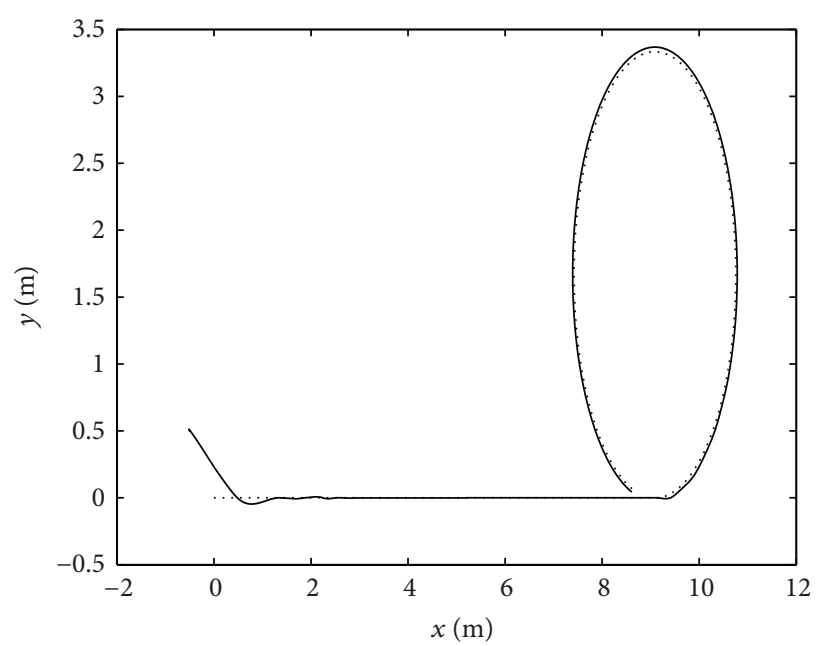

- Actual path ..... Reference path

(a)

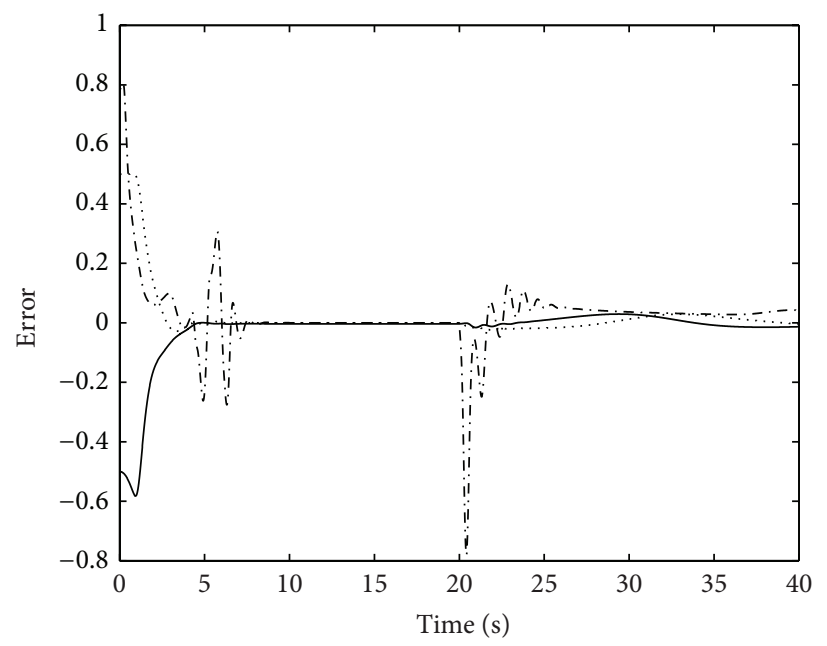

(c)

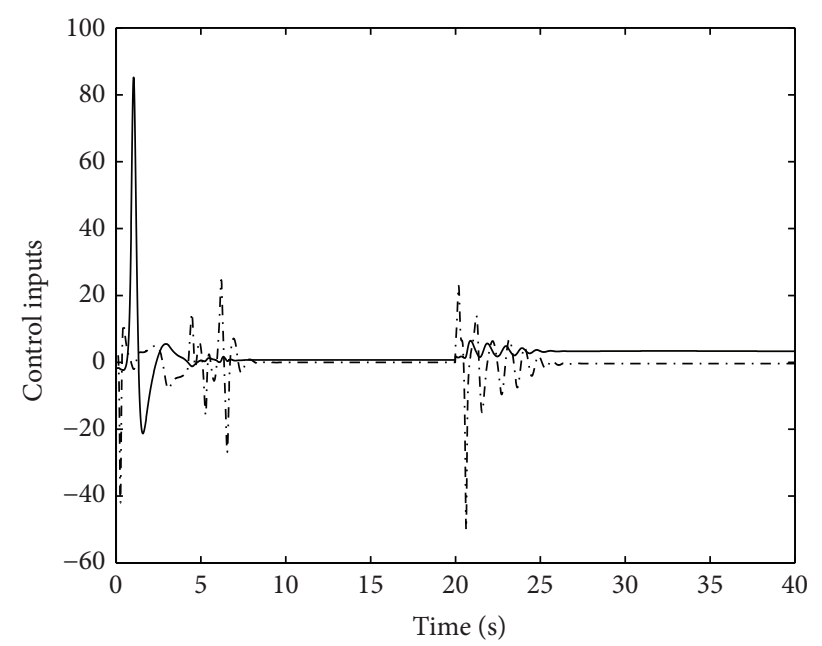

(b)

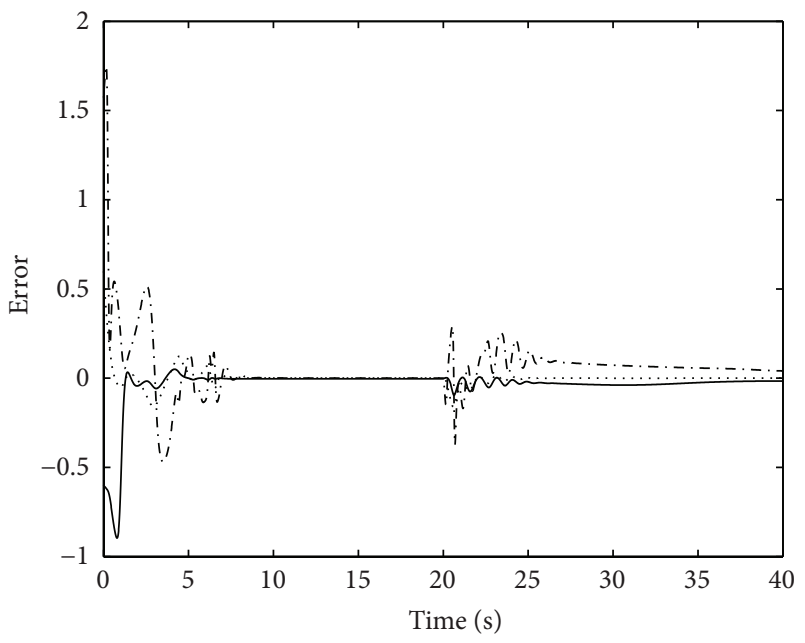

(d)

FIGURE 1: Simulation results. (a) Trajectory tracking result. (b) Control inputs (solid: $\bar{\tau}_{1}$, dash-dot: $\bar{\tau}_{2}$ ). (c) Tracking errors (solid: $e_{1}$, dotted: $e_{2}$, and dash-dot: $e_{3}$ ). (d) Tracking errors (solid: $e_{4}$, dotted: $e_{5}$, and dash-dot: $e_{6}$ ).

\section{Appendix}

Proof of Property 1 . Since $\sigma(\cdot)$ and $\bar{\sigma}(\cdot)$ are bounded, the unknown term $P$ in (12) can be written as

$$
\|P\| \leq \alpha_{0}\|\widetilde{W}\|_{F}\|\widetilde{V}\|_{F}\left\|z_{2}\right\|+\|W\|_{F}\left(\alpha_{1}+\|\widetilde{V}\|_{F}\left\|z_{2}\right\|\right)+\|\varepsilon\|,
$$

where $\alpha_{0}$ and $\alpha_{1}$ are positive constants. Using Assumption 2 and triangular inequality, (A.1) is bounded by

$$
\begin{aligned}
\|P\| \leq & \alpha_{0}\left(W_{M}+\|\widehat{W}\|_{F}\right)\left(V_{M}+\|\widehat{V}\|_{F}\right)\left\|z_{2}\right\| \\
& +\alpha_{1} W_{M}+\alpha_{1} W_{M}\left(V_{M}+\|\widehat{V}\|_{F}\right)\left\|z_{2}\right\|+\varepsilon_{M}
\end{aligned}
$$

$$
\begin{aligned}
= & c_{0}+c_{1}\left\|z_{2}\right\|+c_{2}\|\widehat{W}\|_{F}\left\|z_{2}\right\|+c_{3}\|\widehat{V}\|_{F}\left\|z_{2}\right\| \\
& +c_{4}\|\widehat{W}\|_{F}\|\widehat{V}\|_{F}\left\|z_{2}\right\| \\
= & \delta^{T} \Theta
\end{aligned}
$$

where $c_{0}=\alpha_{1} W_{M}\left(1+V_{M}\right)+\varepsilon_{M}, c_{1}=\alpha_{0} W_{M} V_{M}+\alpha_{1} W_{M} V_{M}$, $c_{2}=\alpha_{0} V_{M}, c_{3}=\alpha_{0} W_{M}+\alpha_{1} W_{M}$, and $c_{4}=\alpha_{0}$.

Proof of Property 2. Since $f_{1}$ and $f_{2}$ are bounded from (8) such that $0<f_{1} \leq 1$ and $0<f_{2} \leq 1$, one can easily prove that $0<g_{1}(t) \leq 1$ and $0<g_{2}(t) \leq 1$. 
Proof of Theorem 5. We prove Theorem 5 in two steps. The first step is to prove boundness of $e_{1}$ and $e_{2}$. In the second, we prove boundness of $e_{3}$.

Step 1. The time derivative of $V_{1}$ using (16)-(20) and (23)-(26) results in

$$
\begin{aligned}
\dot{V}_{1}= & -k_{1} e_{1}^{2}+e_{1} \cos z_{13}\left(\omega_{1}+\gamma_{2} \tanh \beta_{1}\right) \\
& -e_{1} \sin z_{13}\left(\omega_{2}+\gamma_{2} \tanh \beta_{2}\right) \\
& -k_{1} e_{2}^{2}+e_{2} \sin z_{13}\left(\omega_{1}+\gamma_{2} \tanh \beta_{1}\right) \\
& +e_{2} \cos z_{13}\left(\omega_{2}+\gamma_{2} \tanh \beta_{2}\right) \\
& -k_{3} e_{4}^{2}+g_{1}(t) N\left(\zeta_{1}\right) \dot{\zeta}_{1}+\dot{\zeta}_{1}-\widehat{\delta}_{1}^{T} \Theta_{1}\left|e_{4}\right| \\
& +e_{4}\left(\widetilde{W}_{1}^{T} \sigma\left(\widehat{V}_{1}^{T} z_{2}\right)+\widehat{W}_{1}^{T} \bar{\sigma}\left(\widehat{V}_{1}^{T} z_{2}\right) \widetilde{V}_{1}^{T} z_{2}+P_{1}\right) \\
& -k_{3} e_{5}^{2}-\widehat{\delta}_{2}^{T} \Theta_{2}\left|e_{5}\right| \\
& +e_{5}\left(\widetilde{W}_{2}^{T} \sigma\left(\widehat{V}_{2}^{T} z_{2}\right)+\widehat{W}_{2}^{T} \bar{\sigma}\left(\widehat{V}_{2}^{T} z_{2}\right) \widetilde{V}_{2}^{T} z_{2}+P_{2}\right) \\
& -\left(\frac{\omega_{1}^{2}}{\kappa_{1}}+\widehat{\omega}_{1} \Upsilon_{1}\right)-\left(\frac{\omega_{2}^{2}}{\kappa_{2}}+\oplus_{2} \Upsilon_{2}\right) \\
& -\sum_{i=1}^{2}\left(\widetilde{W}_{i}^{T} \Lambda_{i}^{-1} \dot{\hat{W}}_{i}+\widetilde{V}_{i}^{T} \Lambda_{i}^{-1} \dot{\widehat{V}_{i}}+\widetilde{\delta}_{i}^{T} \Lambda_{i}^{-1} \dot{\widehat{\delta}}_{i}\right) \\
&
\end{aligned}
$$

where

$$
\begin{aligned}
& \Upsilon_{1}(\left.e_{1}, e_{2}, e_{3}, e_{4}, e_{6}, \omega_{1}, \omega_{3}, u_{d}, \bar{v}_{d}, r_{d}, \psi_{d}, \dot{u}_{d}, \dot{\bar{v}}_{d}, k_{1}, \gamma_{2}\right) \\
&=-k_{1} \dot{e}_{1} \cos z_{13}+k_{1} e_{1} z_{23} \sin z_{13}-k_{1} \dot{e}_{2} \sin z_{13} \\
& \quad-k_{1} e_{2} z_{23} \cos z_{23}+\cos \left(z_{13}-\psi_{d}\right)\left(\dot{u}_{d}+\bar{v}_{d}\left(z_{23}-r_{d}\right)\right) \\
&+\sin \left(z_{13}-\psi_{d}\right)\left(\dot{\bar{v}}_{d}-u_{d}\left(z_{23}-r_{d}\right)\right) \\
& \Upsilon_{2}\left(e_{1}, e_{2}, e_{3}, e_{5}, e_{6}, \omega_{2}, \omega_{3}, u_{d}, \bar{v}_{d}, r_{d}, \psi_{d}, \dot{u}_{d}, \dot{\bar{v}}_{d}, k_{1}, \gamma_{2}\right) \\
&=k_{1} \dot{e}_{1} \sin z_{13}+k_{1} e_{1} z_{23} \cos z_{13}-k_{1} \dot{e}_{2} \cos z_{13} \\
& \quad+k_{1} e_{2} z_{23} \sin z_{23}+\cos \left(z_{13}-\psi_{d}\right)\left(\dot{\bar{v}}_{d}-u_{d}\left(z_{23}-r_{d}\right)\right) \\
& \quad-\sin \left(z_{13}-\psi_{d}\right)\left(\dot{u}_{d}+\bar{v}_{d}\left(z_{23}-r_{d}\right)\right) .
\end{aligned}
$$

On $V_{1}=\mu_{1}$ and $V_{2}=\mu_{2}, \Upsilon_{1} \leq \Upsilon_{1, M}$ and $\Upsilon_{2} \leq \Upsilon_{2, M}$, where $\Upsilon_{1, M}$ and $\Upsilon_{2, M}$ are positive constants. Therefore, using (28) and Young's inequality, (A.3) is written as

$$
\begin{aligned}
\dot{V}_{1} \leq & -\left(k_{1}-1\right) e_{1}^{2}-\left(k_{1}-1\right) e_{2}^{2}-k_{3} e_{4}^{2}-k_{3} e_{5}^{2} \\
& -\left(\frac{1}{\kappa_{1}}-\frac{5}{2}\right) \omega_{1}^{2}-\left(\frac{1}{\kappa_{2}}-\frac{5}{2}\right) \omega_{2}^{2} \\
& -\frac{1}{2} \sum_{i=1}^{2} \iota_{i}\left(\widetilde{W}_{i}^{T} \widetilde{W}_{i}+\widetilde{V}_{i}^{T} \widetilde{V}_{i}+\widetilde{\delta}_{i}^{T} \widetilde{\delta}_{i}\right) \\
& +g_{1}(t) N\left(\zeta_{1}\right) \dot{\zeta}_{1}+\dot{\zeta}_{1}+\sum_{i=1}^{2} \frac{\iota_{i}}{2}\left(W_{i, M}^{2}+V_{i, M}^{2}+\delta_{i, M}^{2}\right) \\
& +4 \gamma_{2}^{2}+\frac{1}{2}\left(\Upsilon_{1, M}^{2}+\Upsilon_{2, M}^{2}\right) .
\end{aligned}
$$

Choosing $k_{1}=1+k_{1}^{*}, 1 / \kappa_{1}=(5 / 2)+\kappa_{1}^{*}$, and $1 / \kappa_{2}=(5 / 2)+\kappa_{2}^{*}$ with $k_{1}^{*}, \kappa_{1}^{*}, \kappa_{2}^{*}>0$ yields

$$
\dot{V}_{1} \leq-\varsigma_{1} V_{1}+g_{1}(t) N\left(\zeta_{1}\right) \dot{\zeta}_{1}+\dot{\zeta}_{1}+\varsigma_{2}
$$

where $\varsigma_{1}=\min \left[k_{1}^{*}, k_{3}, \kappa_{1}^{*}, \kappa_{2}^{*}, \iota_{1}, \iota_{2}\right]$ and $\varsigma_{2}=\sum_{i=1}^{2}\left(\iota_{i} / 2\right)$ $\left(W_{i, M}^{2}+V_{i, M}^{2}+\delta_{i, M}^{2}\right)+4 \gamma_{2}^{2}+(1 / 2)\left(\Upsilon_{1, M}^{2}+\Upsilon_{2, M}^{2}\right)$. Upon multiplication of (A.6) by $e^{\varsigma_{1} t}$, it becomes

$$
\frac{d}{d t}\left(V_{1} e^{\varsigma_{1} t}\right) \leq g_{1}(t) N\left(\zeta_{1}\right) \dot{\zeta}_{1} e^{\varsigma_{1} t}+\dot{\zeta}_{1} e^{\varsigma_{1} t}+\varsigma_{2} e^{\varsigma_{1} t}
$$

Integrating it over $[0, t]$, we have

$$
V_{1}(t) \leq \varsigma_{3}+e^{-\varsigma_{1} t} \int_{0}^{t} g_{1} N\left(\zeta_{1}\right) \dot{\zeta}_{1} d \lambda+e^{-\varsigma_{1} t} \int_{0}^{t} \dot{\zeta}_{1} e^{\varsigma_{1} \lambda} d \lambda
$$

where $\varsigma_{3}=\sup _{\lambda \in[0, t]}\left[V_{1}(0) e^{-\varsigma_{1} \lambda}+\left(\varsigma_{2} / \varsigma_{1}\right)\left(1-e^{-\varsigma_{1} \lambda}\right)\right]$. Therefore, we can conclude that $V_{1}(t), \zeta_{1}$, and $\int_{0}^{t} g_{1} N\left(\zeta_{1}\right) \dot{\zeta}_{1}$ are bounded on $\left[0, t_{f}\right)$ according to Lemma 2 .

Step 2. The time derivative of $V_{2}$ using (16)-(20) and (23)-(26) results in

$$
\begin{aligned}
\dot{V}_{2}= & -k_{2} e_{3}^{2}+e_{3}\left(\omega_{3}+\gamma_{2} \tanh \beta_{3}\right)-k_{3} e_{6}^{2}+g_{2}(t) N\left(\zeta_{2}\right) \dot{\zeta}_{2} \\
& +\dot{\zeta}_{2}-\widehat{\delta}_{3}^{T} \Theta_{3}\left|e_{6}\right| \\
& +e_{6}\left(\widetilde{W}_{3}^{T} \sigma\left(\widehat{V}_{3}^{T} z_{2}\right)+\widehat{W}_{3}^{T} \varsigma\left(\widehat{V}_{3}^{T} z_{2}\right) \widetilde{V}_{3}^{T} z_{2}+P_{3}\right) \\
& -\left(\frac{\omega_{3}^{2}}{\kappa_{3}}+\omega_{3} \Upsilon_{3}\right)-\widetilde{W}_{3}^{T} \Lambda_{3}^{-1} \widehat{W}_{3} \\
& -\widetilde{V}_{3}^{T} \Lambda_{3}^{-1} \dot{\widehat{V}}_{3}-\widetilde{\delta}_{3}^{T} \Lambda_{3}^{-1} \dot{\widehat{\delta}}_{3},
\end{aligned}
$$

where

$$
\begin{aligned}
& \Upsilon_{3}\left(e_{3}, e_{4}, e_{5}, e_{6}, \omega_{1}, \omega_{2}, \omega_{3}, u_{d}, \bar{v}_{d}, r_{d}, \dot{u}_{d}, \dot{\bar{v}}_{d}, k_{2}, \gamma_{2}\right) \\
& \quad=-k_{2} \dot{e}_{3}+\ddot{\psi}_{a} .
\end{aligned}
$$


On $V_{1}=\mu_{1}$ and $V_{2}=\mu_{2}, \Upsilon_{3} \leq \Upsilon_{3, M}$, where $\Upsilon_{3, M}$ is a positive constant. Then, using (28) and Young's inequality, (A.3) is written as

$$
\begin{aligned}
\dot{V}_{2} \leq & -\left(k_{2}-1\right) e_{3}^{2}-k_{3} e_{6}^{2}-\left(\frac{1}{\kappa_{3}}-1\right) \omega_{3}^{2}+g_{2}(t) N\left(\zeta_{2}\right) \dot{\zeta}_{2} \\
& +\dot{\zeta}_{2}-\frac{\iota_{3}}{2}\left(\widetilde{W}_{3}^{T} \widetilde{W}_{3}+\widetilde{V}_{3}^{T} \widetilde{V}_{3}+\widetilde{\delta}_{3}^{T} \widetilde{\delta}_{3}\right) \\
& +\frac{1}{2}\left(\gamma_{2}^{2}+\iota_{3}\left(W_{3, M}^{2}+V_{3, M}^{2}+\delta_{3, M}^{2}\right)+\Upsilon_{3, M}^{2}\right) .
\end{aligned}
$$

Choosing $k_{2}=1+k_{2}^{*}$ and $1 / \kappa_{3}=1+\kappa_{3}^{*}$ with $k_{2}^{*}, \kappa_{3}^{*}>0$ yields

$$
\dot{V}_{2} \leq-\varsigma_{4} V_{2}+g_{2}(t) N\left(\zeta_{2}\right) \dot{\zeta}_{2}+\dot{\zeta}_{2}+\varsigma_{5}
$$

where $\varsigma_{4}=\min \left[k_{2}^{*}, k_{3}, \kappa_{3}^{*}, \iota_{3}\right]$ and $\varsigma_{5}=(1 / 2)\left(\gamma_{2}^{2}+\iota_{3}\left(W_{3, M}^{2}+\right.\right.$ $\left.\left.V_{3, M}^{2}+\delta_{3, M}^{2}\right)+\Upsilon_{3, M}^{2}\right)$. Similarly, we have

$$
V_{2}(t) \leq \varsigma_{6}+e^{-\varsigma_{4} t} \int_{0}^{t} g_{2} N\left(\zeta_{2}\right) \dot{\zeta}_{2} e^{\varsigma_{4} \lambda} d \lambda+e^{-\varsigma_{4} t} \int_{0}^{t} \dot{\zeta}_{2} e^{\varsigma_{4} \lambda} d \lambda
$$

where $\varsigma_{6}=\sup _{\lambda \in[0, t]}\left[V_{2}(0) e^{-\varsigma_{4} \lambda}+\left(\varsigma_{5} / \varsigma_{4}\right)\left(1-e^{-\varsigma_{4} \lambda}\right)\right]$. Therefore, we can conclude that $V_{2}(t), \zeta_{2}$, and $\int_{0}^{t} g_{2} N\left(\zeta_{2}\right) \dot{\zeta}_{2}$ are bounded on $\left[0, t_{f}\right)$ according to Lemma 2.

Since $V_{1}$ and $V_{2}$ are bounded, one can easily prove from (25) that $\dot{\zeta}_{1}$ and $\dot{\zeta}_{2}$ are bounded. Then, $g_{1}(t) N\left(\zeta_{1}\right) \dot{\zeta}_{1}+\dot{\zeta}_{1}$ and $g_{2}(t) N\left(\zeta_{2}\right) \dot{\zeta}_{2}+\dot{\zeta}_{2}$ are bounded such that $\left|g_{1}(t) N\left(\zeta_{1}\right) \dot{\zeta}_{1}+\dot{\zeta}_{1}\right| \leq$ $\varsigma_{7}$ and $\left|g_{2}(t) N\left(\zeta_{2}\right) \dot{\zeta}_{2}+\dot{\zeta}_{2}\right| \leq \varsigma_{8}$, where $\varsigma_{7}$ and $\varsigma_{8}$ are positive constants. From (A.6) and (A.12), $\dot{V}_{1}<0$ on $V_{1}=\mu_{1}$ when $\varsigma_{1}>\left(\varsigma_{2}+\varsigma_{7}\right) / \mu_{1}$, and $\dot{V}_{2}<0$ on $V_{2}=\mu_{2}$ when $\varsigma_{4}>\left(\varsigma_{5}+\right.$ $\left.\varsigma_{8}\right) / \mu_{2}$. This means that $V_{1} \leq \mu_{1}$ and $V_{2} \leq \mu_{2}$ are invariant sets. Moreover, the boundedness of $V_{1}$ and $V_{2}$ implies that $e_{1}$, $e_{2}$, and $e_{3}$ are bounded. Therefore, we can conclude from (4) and (15) that the inequality in (29) holds.

Remark A.1. The choice of control gains has some suggestions as follows: (i) increasing $k_{1}, k_{3}, \iota_{1}$, and $\iota_{2}$ and decreasing $\kappa_{1}$ and $\kappa_{2}$ help to increase $\varsigma_{1}$, subsequently reducing the bound $\varsigma_{2} / \varsigma_{1}$ of error; (ii) increasing $k_{2}, k_{3}$, and $\iota_{3}$ and decreasing $\kappa_{3}$ help to increase $\varsigma_{4}$, subsequently reducing the bound $\varsigma_{5} / \varsigma_{4}$ of error.

\section{Conflict of Interests}

The author declares that there is no conflict of interests regarding the publication of this paper.

\section{Acknowledgment}

This work was supported by Basic Science Research Program through the National Research Foundation of Korea (NRF) funded by the Ministry of Education, Science and Technology (NRF-2012R1A1A1041216).

\section{References}

[1] M. L. Corradini, A. Monteriu, G. Orlando, and S. Pettinari, "An actuator failure tolerant robust control approach for an underwater remotely operated vehicle," in Proceedings of the 50th IEEE Conference on Decision and Control and European Control Conference, pp. 3934-3939, Orlando, Fla, USA, December 2011.

[2] A. Alessandri, M. Caccia, and G. Veruggio, "Fault detection of actuator faults in unmanned underwater vehicles," Control Engineering Practice, vol. 7, no. 3, pp. 357-368, 1999.

[3] M. Blanke, M. Staroswiecki, and N. E. Wu, "Concepts and methods in fault-tolerant control," in Proceedings of the American Control Conference (ACC '01), pp. 2606-2620, Arlington, Va, USA, June 2001.

[4] R. Patton, "Fault-tolerant control systems: the 1997 situation," in Proceedings of the IFAC Symposium on Fault Detection Supervision and Safety for Technical Processes, pp. 1033-1054, Brussels, Belgium, 1997.

[5] N. Sarkar, T. K. Podder, and G. Antonelli, "Faultaccommodating thruster force allocation of an AUV considering thruster redundancy and saturation," IEEE Transactions on Robotics and Automation, vol. 18, no. 2, pp. 223-233, 2002.

[6] T. Perez and A. Donaire, "Constrained control design for dynamic positioning of marine vehicles with control allocation," Modeling, Identification and Control, vol. 30, no. 2, pp. 57-70, 2009.

[7] E. Omerdic and G. Roberts, "Thruster fault diagnosis and accommodation for open-frame underwater vehicles," Control Engineering Practice, vol. 12, no. 12, pp. 1575-1598, 2004.

[8] B. Sun, D. Zhu, and L. Sun, "A tracking control method with thruster fault tolerant control for unmanned underwater vehicles," in Proceedings of the 25th Chinese Control and Decision Conference (CCDC '13), pp. 4915-4920, Guiyang, China, May 2013.

[9] X. Chen and W. W. Tan, "Tracking control of surface vessels via fault-tolerant adaptive backstepping interval type-2 fuzzy control," Ocean Engineering, vol. 70, pp. 97-109, 2013.

[10] G. J. Toussaint, T. Basar, and F. Bullo, "Tracking for nonlinear underactuated surface vessels with generalized forces," in Proceedings of the IEEE International Conference on Control Applications, pp. 355-360, Anchorage, Alaska, USA, April 2000.

[11] C. Kwan, D. M. Dawson, and F. L. Lewis, "Robust adaptive control of robots using neural network: global stability," Asian Journal of Control, vol. 3, no. 2, pp. 111-121, 2001.

[12] S. N. Huang, K. K. Tan, and T. H. Lee, "Automated fault detection and diagnosis in mechanical systems," IEEE Transactions on Systems, Man and Cybernetics Part C: Applications and Reviews, vol. 37, no. 6, pp. 1360-1364, 2007.

[13] S. N. Huang and K. K. Tan, "Fault detection, isolation, and accommodation control in robotic systems," IEEE Transactions on Automation Science and Engineering, vol. 5, no. 3, pp. 480489, 2008.

[14] F. Caccavale, P. Cilibrizzi, F. Pierri, and L. Villani, "Actuators fault diagnosis for robot manipulators with uncertain model," Control Engineering Practice, vol. 17, no. 1, pp. 146-157, 2009.

[15] R. D. Nussbaum, "Some remarks on a conjecture in parameter adaptive control," Systems \& Control Letters, vol. 3, no. 5, pp. 243-246, 1983

[16] T. I. Fossen, Marine Control Systems, Marine Cybernetics, Trondheim, Norway, 2002. 
[17] K. D. Do and J. Pan, "Global tracking control of underactuated ships with nonzero off-diagonal terms in their system matrices," Automatica, vol. 41, no. 1, pp. 87-95, 2005.

[18] Y. Zhang, L. Wu, and S. Wang, "UCAV path planning based on FSCABC," Information, vol. 14, no. 3, pp. 687-692, 2011.

[19] Y. Zhang, L. Wu, and S. Wang, "UCAV path planning by fitness-scaling adaptive chaotic particle swarm optimization," Mathematical Problems in Engineering, vol. 2013, Article ID 705238, 9 pages, 2013.

[20] F. L. Lewis, S. Jagannathan, and A. Yesilderek, Neural Network Control of Robot Manipulator and Nonlinear Systems, Taylor \& Francis, London, UK, 1999.

[21] S. S. Ge and J. Wang, "Robust adaptive tracking for timevarying uncertain nonlinear systems with unknown control coefficients," IEEE Transactions on Automatic Control, vol. 48, no. 8, pp. 1463-1469, 2003.

[22] R. Skjetne, T. I. Fossen, and P. Kokotovic, "Adaptive maneuvering, with experiments, for a model ship in a marine control laboratory," Automatica, vol. 41, no. 2, pp. 289-298, 2005. 


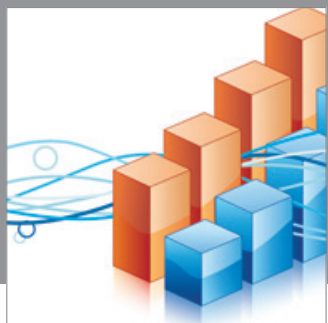

Advances in

Operations Research

mansans

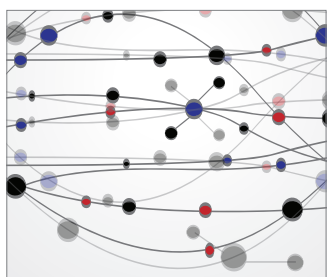

The Scientific World Journal
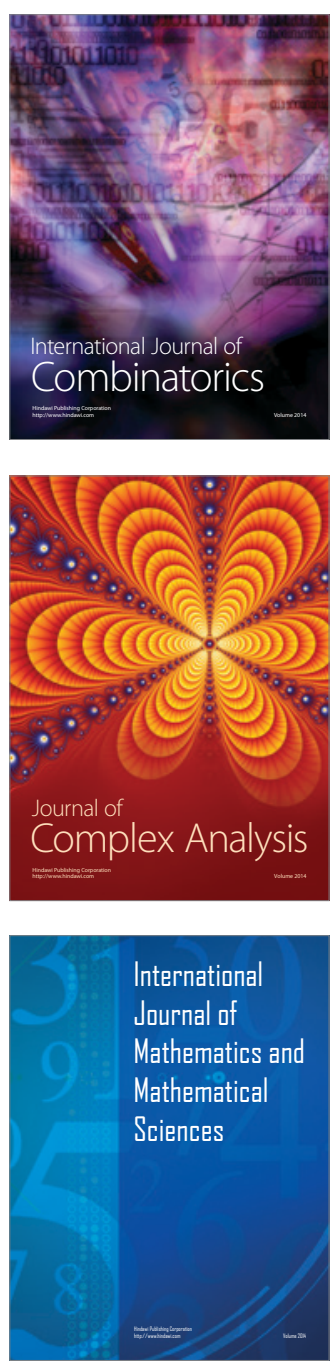
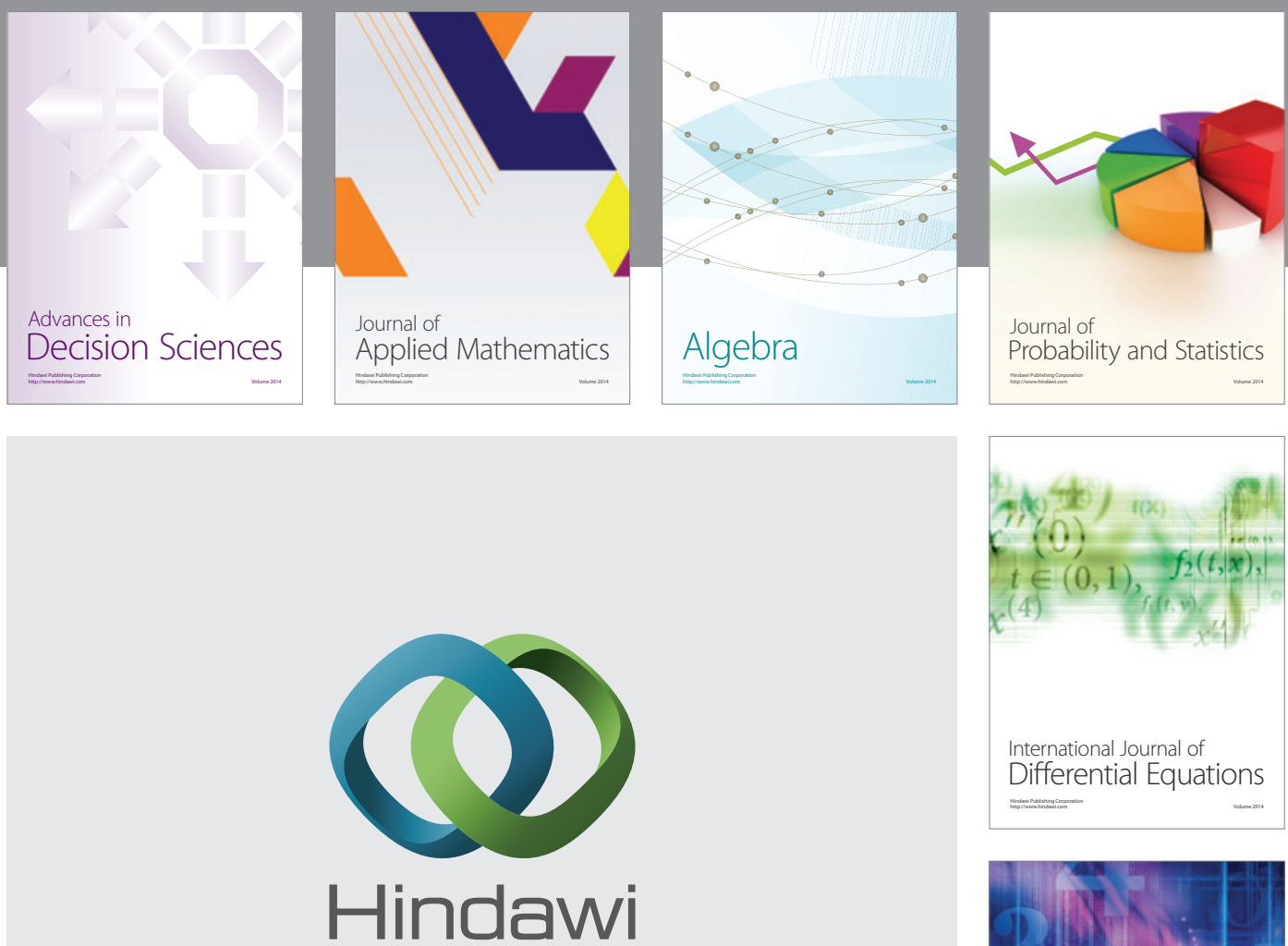

Submit your manuscripts at http://www.hindawi.com
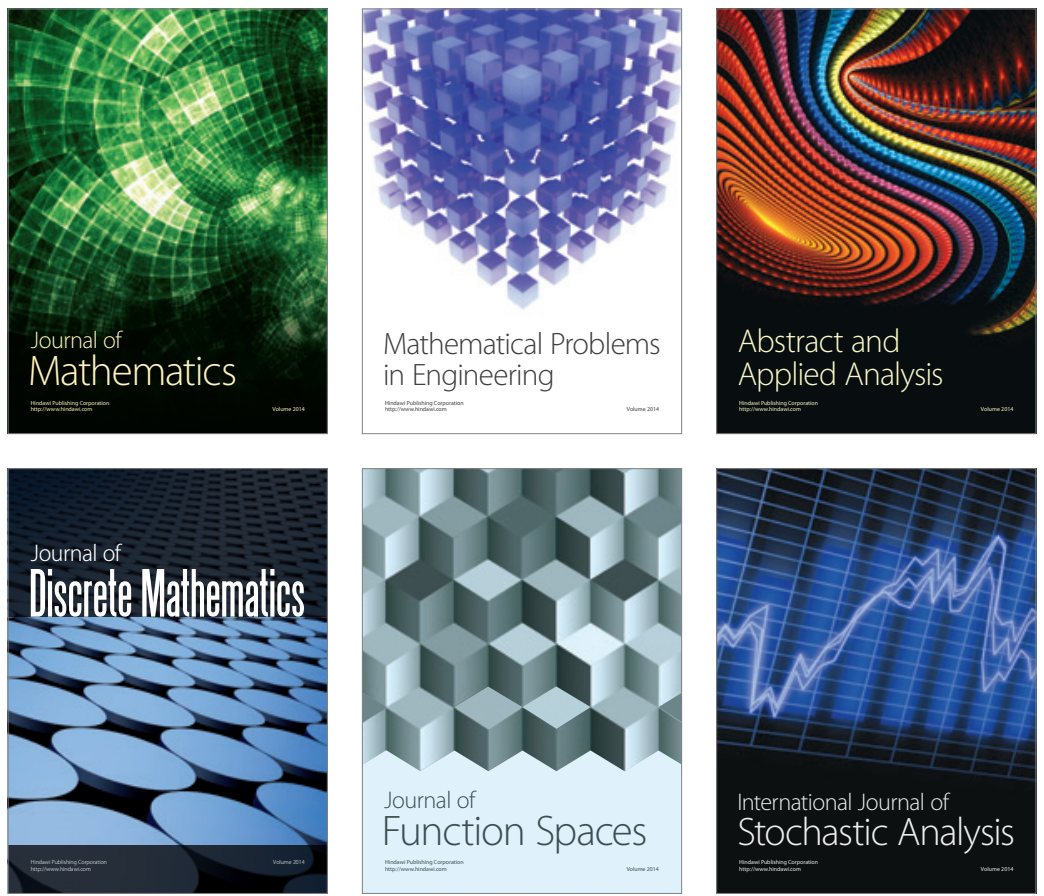

Journal of

Function Spaces

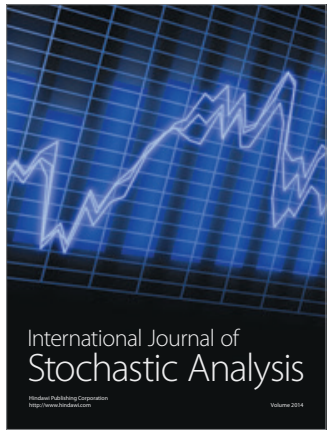

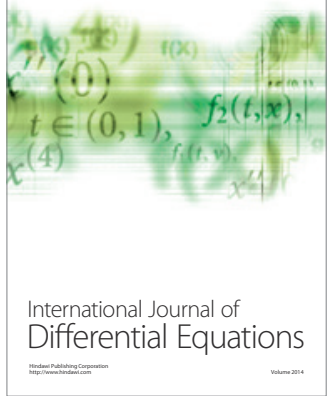
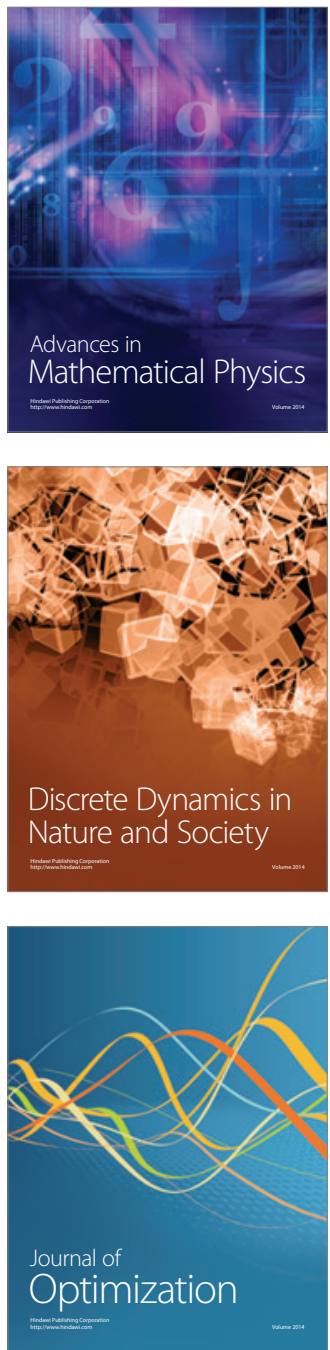